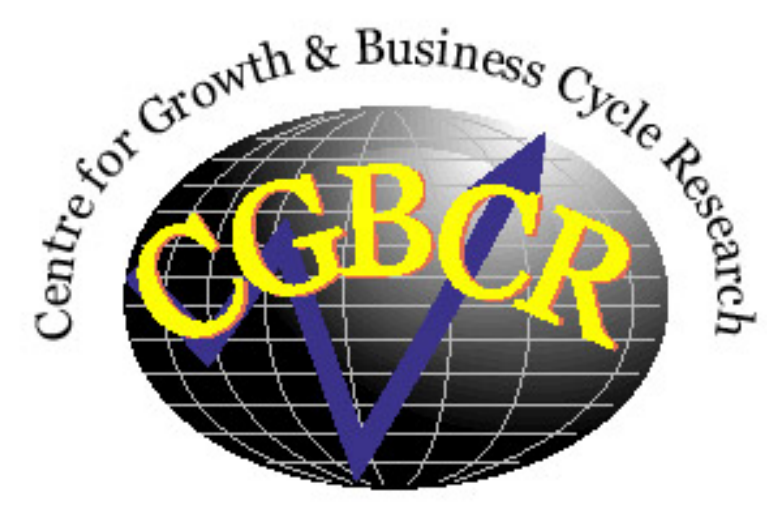

Discussion Paper Series

\title{
Testing for causality in variance in the presence of breaks
}

\author{
By
}

Dick van Dijk ${ }^{*}$, Denise R. Osborn ${ }^{\dagger}$ and Marianne Sensier ${ }^{\dagger}$

*Econometric Institute, Erasmus University Rotterdam

${ }^{\dagger}$ Centre for Growth and Business Cycle Research, Economic Studies,

University of Manchester, Manchester, M13 9PL, UK

November 2004

Number 045

\section{Download paper from:}

http://www.ses.man.ac.uk/cgbcr/discussi.htm 


\title{
Testing for Causality in Variance in the Presence of Breaks*
}

\author{
Dick van Dijk ${ }^{\dagger}$ \\ Econometric Insitute \\ Erasmus University Rotterdam
}

\author{
Denise R. Osborn \\ Centre for Growth and Business Cycle Research \\ Economic Studies \\ University of Manchester \\ Marianne Sensier \\ Centre for Growth and Business Cycle Research \\ Economic Studies \\ University of Manchester
}

November 2004

\begin{abstract}
We examine the size properties of causality-in-variance tests in the presence of structural breaks in volatility. Extensive Monte Carlo simulations demonstrate that these tests suffer from severe size distortions when such breaks are not taken into account. Pre-testing the series for structural changes in volatility is shown to largely remedy the problem.
\end{abstract}

Keywords: volatility; causality tests; structural change.

JEL Classification Codes: C12; C22.

*The second and third authors acknowledge support from the Economic and Social Research Council (UK) under grant number L138251030.

${ }^{\dagger}$ Address for correspondence: Econometric Institute, Erasmus University Rotterdam, P.O. Box 1738, NL-3000 DR Rotterdam, The Netherlands, e-mail: djvandijk@few.eur.nl 


\section{Introduction}

The modelling of volatility spillovers in the finance literature has been important since Morgenstern (1959) and more recently with Lin, Engle and Ito (1994), and Billio and Pelizzon (2003). These papers estimate parametric models to examine specific formulations for the spillover effects, while Cheung and Ng (1996) and Hong (2001) develop general causality-in-variance tests within this framework.

There has recently been increasing awareness that many time series experience occasional structural breaks in (unconditional) volatility; see Andreou and Ghysels (2002), McConnell and Perez-Quiros (2000) and Sensier and van Dijk (2004), among others. Given the evidence for such structural breaks, it is important to investigate whether they affect the appearance of volatility spillover effects. The present paper examines this question by analyzing the impact of volatility breaks on the causality-in-variance tests. Based on an extensive set of Monte Carlo simulations, we demonstrate that these tests suffer from severe size distortions when such breaks are neglected. However, we also show that pre-testing for volatility breaks provides an effective solution to this size problem.

\section{Tests for causality in variance}

Let $y_{t}=\left(y_{1 t}, y_{2 t}\right)^{\prime}$ be a bivariate series of interest. In Cheung and $\mathrm{Ng}$ (1996), $y_{2 t}$ is said to cause $y_{1 t}$ in variance if

$$
\mathrm{E}\left[\left(y_{1 t}-\mu_{1 t}\right)^{2} \mid \Omega_{t-1}\right] \neq \mathrm{E}\left[\left(y_{1 t}-\mu_{1 t}\right)^{2} \mid \Omega_{1 t-1}\right]
$$

where $\Omega_{i t}$ is the information set defined by $\Omega_{i t}=\left\{y_{i t-j} ; j \geq 0\right\}, i=1,2, \Omega_{t}=\Omega_{1 t} \cup \Omega_{2 t}$, and $\mu_{i t}$ is the mean of $y_{i t}$ conditional on $\Omega_{i t}$. Let $\varepsilon_{i t}=y_{i t}-\mu_{i t}, i=1,2$, and assume that $\varepsilon_{i t}=\xi_{i t} \sqrt{h_{i t}}$, where $h_{i t}$ is a positive, time-varying function measurable with respect to $\Omega_{i t-1}$, and $\xi_{i t}$ is an innovation process with $\mathrm{E}\left[\xi_{i t} \mid \Omega_{i t-1}\right]=0$ and $\mathrm{E}\left[\xi_{i t}^{2} \mid \Omega_{i t-1}\right]=1$. Hence $h_{i t}$ is the (univariate) conditional variance of $\varepsilon_{i t}$ by construction, that is $h_{i t}=\mathrm{V}\left[\varepsilon_{i t} \mid \Omega_{i t-1}\right]$. 
The null hypothesis that $y_{2 t}$ does not cause $y_{1 t}$ in variance can now be formulated as

$$
\mathrm{H}_{0}: \mathrm{V}\left[\xi_{1 t} \mid \Omega_{t-1}\right]=\mathrm{V}\left[\xi_{1 t} \mid \Omega_{1 t-1}\right]
$$

Define the squared standardized residuals

$$
u_{t}=\left(y_{1 t}-\hat{\mu}_{1 t}\right)^{2} / \hat{h}_{1 t} \quad \text { and } \quad v_{t}=\left(y_{2 t}-\hat{\mu}_{2 t}\right)^{2} / \hat{h}_{2 t}
$$

where hats indicate suitable estimates of the corresponding quantities, and the sample cross-correlation at lag $k$,

$$
r_{u v}(k)=c_{u v}(k) / \sqrt{c_{u u}(0) c_{v v}(0)}
$$

where $c_{u v}(k)$ is the sample cross-covariance

$$
c_{u v}(k)= \begin{cases}\frac{1}{T} \sum_{t=k+1}^{T}\left(u_{t}-\bar{u}\right)\left(v_{t-k}-\bar{v}\right), & \text { if } k \geq 0 \\ \frac{1}{T} \sum_{t=1}^{T-k}\left(u_{t}-\bar{u}\right)\left(v_{t-k}-\bar{v}\right), & \text { if } k<0,\end{cases}
$$

with $T$ denoting sample size, $\bar{u}$ and $\bar{v}$ the sample means of $u_{t}$ and $v_{t}$, respectively, and $c_{x x}(0)=1 / T \sum_{t=1}^{T}\left(x_{t}-\bar{x}\right)^{2}$ for $x=u, v$.

Cheung and $\mathrm{Ng}(1996)$ suggest testing $H_{0}$ using the statistic

$$
S=T \sum_{k=1}^{K} r_{u v}^{2}(k)
$$

which has an asymptotic chi-square distribution with $K$ degrees of freedom. The reverse hypothesis that $y_{1 t}$ does not cause $y_{2 t}$ in variance can be tested analogously, summing the squared cross-correlations $r_{u v}^{2}(k)$ from $k=-K$ to -1 .

Hong (2001) modifies the Cheung-Ng test in two ways. First, $\mu_{i t}, i=1,2$, is defined to be the mean of $y_{i t}$ conditional on the complete "bivariate" information set $\Omega_{t-1}$. This ensures that any causality-in-mean is filtered out when testing for causality-in-variance. Pantelidis and Pittis (2004) show that this is important, as neglected causality-in-mean leads to severe size distortions for the Cheung-Ng test. Second, Hong (2001) suggests weighting the cross-correlations to obtain more powerful tests as follows:

$$
Q=\frac{T \sum_{k=1}^{T-1} w^{2}(k ; K) r_{u v}^{2}(k)-C(w)}{\sqrt{2 D(w)}} \stackrel{\text { asy }}{\sim} N(0,1),
$$


where $w(k ; K)$ is a weight function, for which we use the Bartlett kernel

$$
w(k ; K)= \begin{cases}1-|k /(K+1)| & \text { if } k /(K+1) \leq 1 \\ 0 & \text { otherwise }\end{cases}
$$

and

$$
\begin{aligned}
C(w) & =\sum_{k=1}^{T-1}(1-k / T) w^{2}(k ; K), \\
D(w) & =\sum_{k=1}^{T-1}(1-k / T)(1-(k+1) / T) w^{4}(k ; K),
\end{aligned}
$$

are approximately the mean and variance, respectively, of $T \sum_{k=1}^{T-1} w^{2}(k / K) r_{u v}^{2}(k)$.

\section{Monte Carlo design and size results}

We examine the size of causality-in-variance tests for a data generating process with $y_{t}=\left(y_{1 t}, y_{2 t}\right)^{\prime} \sim N I D\left(0, \Sigma_{t}\right)$, where $\Sigma_{t}=\left(\begin{array}{cc}\sigma_{1 t}^{2} & \sigma_{1 t} \sigma_{2 t} \rho \\ \sigma_{1 t} \sigma_{2 t} \rho & \sigma_{2 t}^{2}\end{array}\right)$. The effects of neglected changes in volatility are examined through the following five experiments: ${ }^{1}$

A. Shift in volatility of $y_{2 t}$ only: $\sigma_{1 t}^{2}=1$ for all $t$, while $\sigma_{2 t}^{2}=\sigma_{b}^{2}$ for $t \leq T / 2$ and $\sigma_{2 t}^{2}=\sigma_{a}^{2}$ for $t>T / 2$

B. Simultaneous and identical changes in volatility: $\sigma_{1 t}^{2}=\sigma_{2 t}^{2}=\sigma_{b}^{2}$ for $t \leq T / 2$ and $\sigma_{1 t}^{2}=\sigma_{2 t}^{2}=\sigma_{a}^{2}$ for $t>T / 2$

C. Simultaneous but opposite changes in volatility: $\sigma_{1 t}^{2}=\sigma_{a}^{2}, \sigma_{2 t}^{2}=\sigma_{b}^{2}$ for $t \leq T / 2$, and $\sigma_{1 t}^{2}=\sigma_{b}^{2}, \sigma_{2 t}^{2}=\sigma_{a}^{2}$ for $t>T / 2$;

D. Simultaneous decline in volatility: $\sigma_{2 t}^{2}$ and $\sigma_{1 t}^{2}$ change from 1 to 0.5 at $t=\pi T$;

E. Declines at different times: $\sigma_{1 t}^{2}$ changes at $t=T / 2$ while $\sigma_{2 t}^{2}$ changes at $t=\pi T$, both from 1 to 0.5 .

\footnotetext{
${ }^{1}$ Analogously to experiments D and E, we also examined increases in volatility in $\sigma_{1 t}^{2}$ and $\sigma_{2 t}^{2}$ from 1 to 2. Results for these are the mirror images of the corresponding cases shown. These results are available on request.
} 
Experiments A to $\mathrm{C}$ use $\sigma_{b}^{2}=1$ and $\sigma_{a}^{2}$ such that $\frac{\sigma_{a}^{2}}{\sigma_{b}^{2}}=0.1,0.2, \ldots 0.9,1.0, \frac{1}{0.9}, \ldots, \frac{1}{0.1}$. Experiments D and E use $\pi=0.10,0.15, \ldots, 0.90$ with $\pi=0.45,0.46, \ldots, 0.55$ also examined for E).

We consider sample sizes of $T=160$ and 480, corresponding to 40 years of quarterly and monthly data, respectively; 10000 replications are used throughout. The test statistics $S$ in (5) and $Q$ in (6) for testing causality-in-variance from $y_{2 t}$ to $y_{1 t}$ and vice versa are computed for $K=1,2, \ldots, 10$, replacing $\hat{\mu}_{i t}$ and $\hat{h}_{i t}$ in $(3)$ by the sample mean and variance, respectively. For space considerations, we only report selected results. Full details are available in an appendix at http://www.ses.man.ac.uk/cgbcr/. Rejection frequencies for the Cheung-Ng test statistic at the nominal $5 \%$ significance level are shown graphically in panel (a) of Figures 1-3.

(Unreported) Results for experiment A show that neglecting structural breaks in volatility has only minor effects when just one of the series experiences a volatility change. In contrast, simultaneous changes in volatility lead to substantially larger size distortions, irrespective of whether the volatility change is identical (Figure 1) or opposite (see the

appendix). In addition, the size distortion is seen to be symmetric in the ratio $\frac{\sigma_{a}^{2}}{\sigma_{b}^{2}}$. Figure 2 makes clear that the timing of the volatility change matters: the size distortions are largest when the simultaneous volatility decline occurs at one-third of the sample period. Finally, from Figure 3 it appears that simultaneous volatility changes lead to the largest size distortions, with the distortion declining as the time interval between breaks increases.

\section{Solving the problem: pre-testing for volatility breaks}

In this section we explore whether pre-testing for structural changes in volatility can remedy the size distortions of the causality-in-variance tests in the presence of such volatility breaks.

As in McConnell and Perez-Quiros (2000), we test for volatility breaks using the absolute values of the demeaned series. We treat the break date $\tau_{i}$ as unknown and use the 
sup-Wald statistic developed by Andrews (1993), given by

$$
\operatorname{SupW}=\sup _{\tau_{\min } \leq \tau_{i} \leq \tau_{\max }} W_{T}\left(\tau_{i}\right)
$$

where $W_{T}\left(\tau_{i}\right)$ denotes the Wald test of the null hypothesis $H_{0}: \delta_{i 1}=\delta_{i 2}$ in the regression

$$
\sqrt{\frac{\pi}{2}}\left|y_{i t}-\hat{\mu}_{i}\right|=\delta_{i 1}\left(1-\mathrm{I}\left(t>\tau_{i}\right)\right)+\delta_{i 2} \mathrm{I}\left(t>\tau_{i}\right)+\varepsilon_{i t}, \quad t=1, \ldots, T
$$

where $\hat{\mu}_{i}$ is the sample mean of $y_{i t}$ and $\mathrm{I}(A)$ is an indicator function for the event $A$. Both pre- and post-break periods are required to contain at least $10 \%$ of the available observations, that is we set $\tau_{\min }=[\pi T]$ and $\tau_{\max }=[(1-\pi) T]+1$ with $\pi=0.10$, where [.] denotes integer part. We use the method of Hansen (1997) to obtain an approximate asymptotic $p$-value of the SupW statistic.

This volatility break test is applied prior to the causality-in-variance test. If, using (7), the null hypothesis of no volatility change in $y_{i t}$ is rejected at a $5 \%$ significance level, we take the estimated volatility break at $\hat{\tau}_{i}$ (the time period that minimizes the sum of squared residuals in (8)) into account when standardizing the series. This is achieved by replacing $\hat{h}_{i t}$ in $(3)$ by the sample variance before (after) $\hat{\tau}_{i}$ for all $t \leq(>) \hat{\tau}_{i}$.

Results are shown in panel (b) of Figures 1-3. In all cases this pre-testing procedure yields empirical rejection frequencies close to the nominal significance level of $5 \%$. For $K= \pm 1$, the procedure tends to yield some under-sizing, with some evidence of over-sizing for larger $K$. Nevertheless, these distortions are relatively modest.

\section{Conclusions}

Since volatility changes have been shown to occur across a wide range of observed economic and financial time series, the severity of the size distortions revealed in our Monte Carlo results appears to indicate that the tests of Cheung and Ng (1996) and Hong (2001) may, in practical applications, provide unreliable inference about the (non-)existence of causality in variance. Size problems arise particularly when both series exhibit volatility changes in close temporal proximity, in which case the tests frequently and incorrectly attribute this 
occurrence to an underlying causality. Pre-testing for structural changes in volatility is shown to remedy this problem. Therefore, we recommend that these causality-in-variance tests should be applied only after such pre-testing for breaks in volatility. 


\section{References}

Andreou, E. and E. Ghysels (2002), Detecting multiple breaks in financial market volatility dynamics, Journal of Applied Econometrics 17, 579-600.

Andrews, D.W.K. (1993), Tests for parameter instability and structural change with unknown change point, Econometrica 61, 821-856.

Billio, M. and L. Pelizzon (2003), Volatility and shocks spillover before and after EMU in European stock markets, Journal of Multinational Financial Management 13, 323-340.

Cheung, Y.-W. and L.K. Ng (1996), A causality-in-variance test and its application to financial market prices, Journal of Econometrics 72, 33-48.

Hansen, B.E. (1997), Approximate asymptotic $p$ values for structural-change tests, Journal of Business and Economic Statistics 15, 60-67.

Hong, Y. (2001), A test for volatility spillover with application to exchange rates, Journal of Econometrics 103, 183-224.

Lin, W.-L., R.F. Engle and T. Ito (1994), Do bulls and bears move across borders? International transmission stock returns and volatility, Review of Financial Studies 7, 507-538.

McConnell, M.M. and G. Perez-Quiros (2000), Output fluctuations in the United States: What has changed since the early 1980s?, American Economic Review 90, 1464-1476.

Morgenstern, O. (1959), International Financial Transactions and the Business Cycle, Princeton, NJ: Princeton University Press.

Pantelidis, T. and N. Pittis (2004), Testing for Granger causality in variance in the presence of causality in mean, Economics Letters 85, 201-207.

Sensier, M. and D. van Dijk (2004), Testing for volatility changes in US macroeconomic time series, Review of Economics and Statistics 86, 833-839. 




(a) Raw series, $T=480$

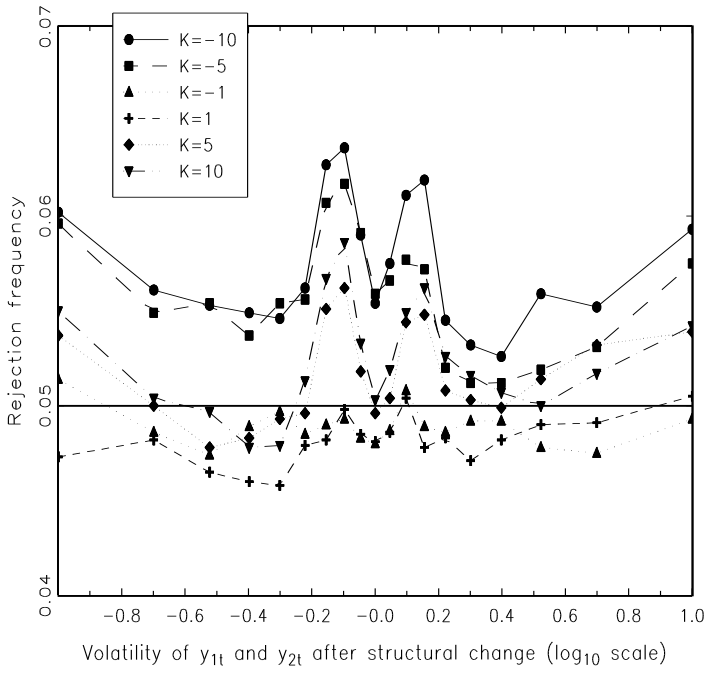

(b) Pre-tested series, $T=480$

Figure 1: Rejection frequencies of the Cheung-Ng test (5) at 5\% nominal significance level for Experiment B.

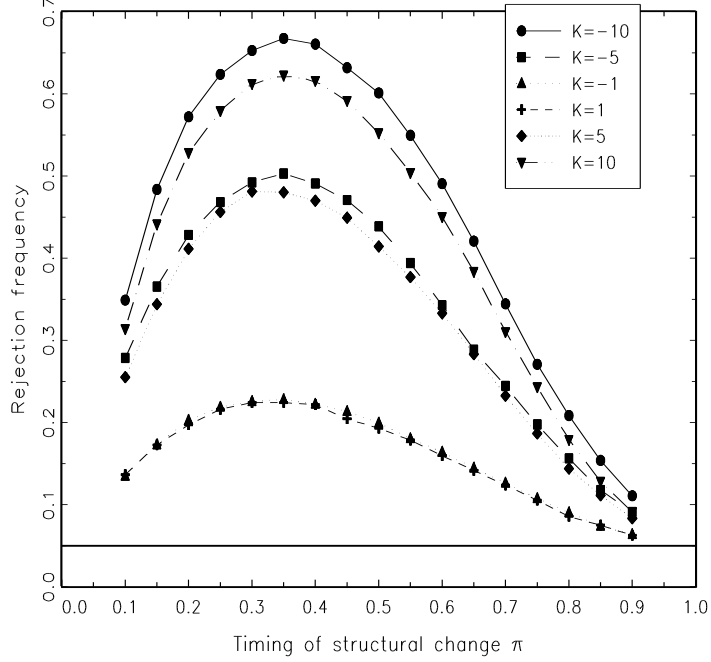

(a) Raw series, $T=480$

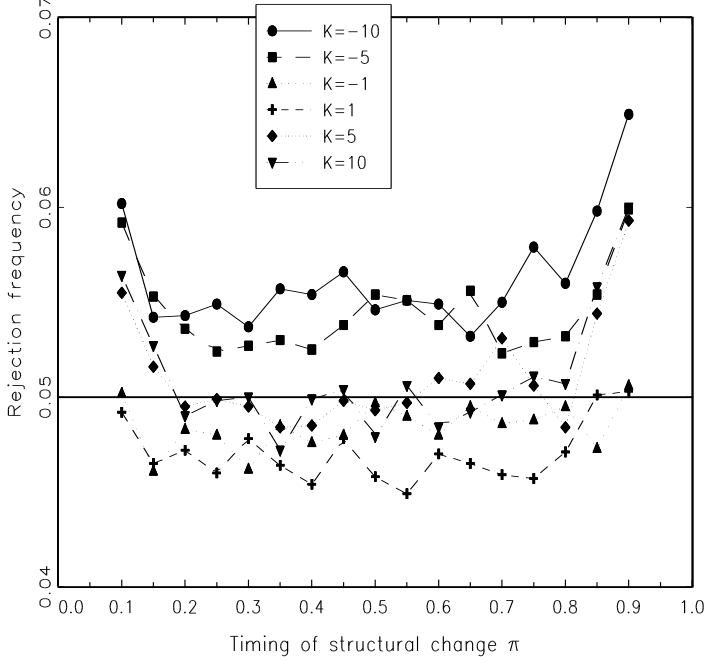

(b) Pre-tested series, $T=480$

Figure 2: Rejection frequencies of the Cheung-Ng test (5) at 5\% nominal significance level for Experiment D. 




(a) Raw series, $T=480$

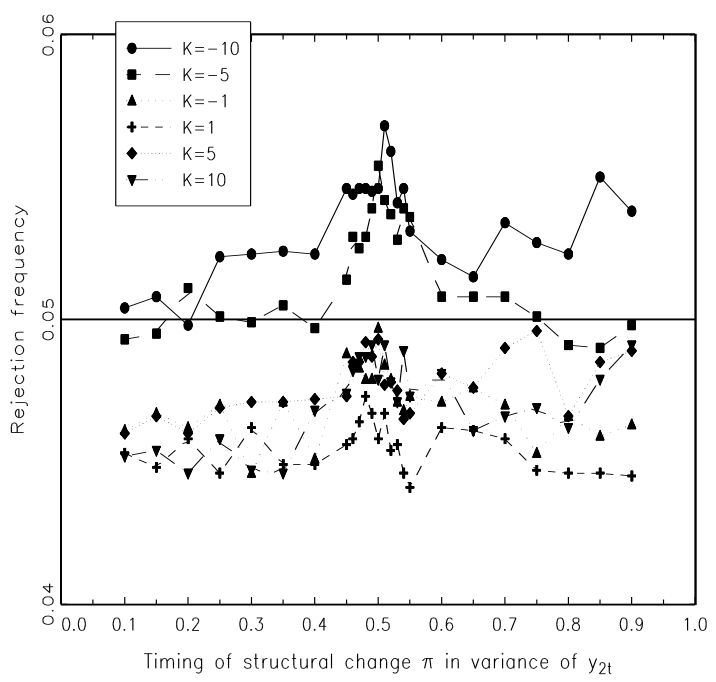

(b) Pre-tested series, $T=480$

Figure 3: Rejection frequencies of the Cheung-Ng test (5) at 5\% nominal significance level for Experiment E. 